\title{
Serviço Computacional para Interpolação de Dados Espaciais
}

\author{
Wellington Luiz Antonio ${ }^{1}$, Diego de Freitas Bezerra ${ }^{1}$, \\ José Clodoalves da Silva Júnior ${ }^{1}$, Victor Wanderley Costa de Medeiros ${ }^{1}$, \\ Glauco Estácio Gonçalves ${ }^{1}$
}

\author{
${ }^{1}$ Departamento de Estatística e Informática - Universidade Federal \\ Rural de Pernambuco, Recife, PE - Brasil \\ \{wellington.luizantonio, diego.freitas, jose.clodoalves, \\ victor.medeiros, glauco.goncalves\}@ufrpe.br
}

\begin{abstract}
Spatial interpolation (IE) allows you to model the surface of a spatially distributed variable from a finite set of data, being important for several areas of expertise. Due to the increasing demand for its use, this work proposes a scalable and versatile web interpolation (SWIE) web service. Results of its performance are also presented and discussed in this work. The main beneficiaries of the web service include researchers and software developers who will use the service to compose software tools that require IE.
\end{abstract}

Resumo. A interpolação espacial (IE) permite modelar a superfície de uma variável espacialmente distribuída a partir de um conjunto finito de dados, sendo importante para diversas áreas de atuação. Devido à demanda cada vez maior pelo seu uso, este trabalho propõe um serviço web de interpolação espacial (SWIE) escalável e versátil. Resultados de seu desempenho também são apresentados e discutidos nesse trabalho. Os principais beneficiários do serviço web incluem pesquisadores e desenvolvedores de software que utilizarão o serviço na composição de ferramentas de software que necessitem da IE.

\section{Introdução}

As técnicas de interpolação espacial (IE) são essenciais na construção de modelos estatístico-computacionais capazes de estimar o valor de uma variável espacialmente distribuída [Jakob and Young 2016]. Tais técnicas fornecem dados que auxiliam a tomada de decisão em aplicações de diversas áreas, tais como: meteorologia [Li and Heap 2014, Lopes et al. 2017], hidrologia [De Vargas et al. 2019], sociologia [Jakob and Young 2016], zoneamento agrícola até a caracterização de áreas de risco à saúde, por exemplo.

Este trabalho apresenta um serviço web de interpolação espacial (SWIE) escalável e versátil. A fim de garantir sua escalabilidade, o serviço foi desenvolvido na forma de uma API REST que pode ser escalada tanto horizontal como verticalmente para atendimento de um maior número de usuários. Com o intuito de validar esta escalabilidade, esse trabalho apresenta uma avaliação de desempenho do serviço sob diferentes condições.

A versatilidade é dada pela ampla gama de variáveis que podem ser interpoladas, tais como temperatura ambiente, umidade relativa do ar, velocidade do vento etc. Além disso, o serviço implementa múltiplos algoritmos para IE, permitindo que o usuário do serviço possa escolher, no momento da geração do modelo, o algoritmo mais adequado para interpolação de sua variável. 
O serviço foi desenvolvido para atuar no contexto de uma arquitetura de microsserviços permitindo que esse seja facilmente integrado a outras ferramentas que necessitem realizar a IE de alguma variável. Atualmente, ele já é utilizado como parte do aplicativo móvel Aquaprev'1. Destinado ao setor agrícola, o Aquaprev interpola espacialmente dados meteorológicos com o objetivo de estimar diariamente o tempo de irrigação de uma determinada cultura.

O trabalho está organizado em cinco Seções. Além desta Seção introdutória, a Seção 2 descreve em detalhes as etapas de especificação, implementação e implantação do serviço web. A Seção 3 apresenta os experimentos realizados para avaliação de desempenho do serviço e a Seção 4 os resultados desta avaliação. Por fim, a Seção 5 apresenta as conclusões decorrentes da análise dos resultados, as principais contribuições e os possíveis trabalhos futuros.

\section{Serviço Web de Interpolação Espacial}

O SWIE baseou-se nos princípios da Arquitetura Orientada a Serviços (Service-Oriented Architecture - SOA). Esta arquitetura estabelece regras e protocolos que permitem o desenvolvimento de serviços interoperáveis que podem ser compartilhados e reutilizados por diferentes aplicações. Estas características são essenciais para o serviço proposto pois permitem o seu desacoplamento dos outros serviços que venham a utilizá-lo.

\subsection{Arquitetura do serviço}

Na Figura 1 é apresentado um exemplo de arquitetura do ambiente, onde o serviço de interpolação espacial poderia estar inserido. O SWIE pode ser consultado por outros componentes de software, tais como, microsserviços para aquisição de dados, ferramentas de análise de dados e aplicativos para usuários finais.

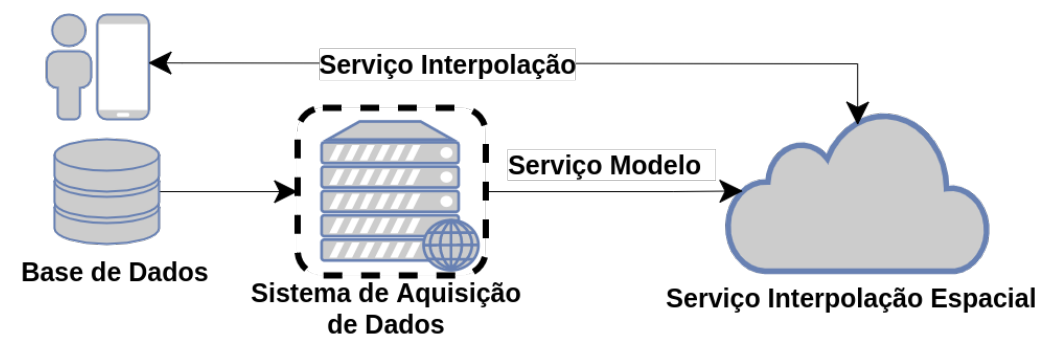

Figura 1. Arquitetura do ambiente onde o SWIE pode ser inserido.

Fonte: Próprio Autor

O SWIE contém dois recursos para a IE: o Model, que cria um modelo de interpolação a partir de um conjunto de observações espaciais; e o Interpolation, que realiza a interpolação propriamente dita para um conjunto de coordenadas fornecidas.

Para o uso do recurso Model (ilustrado na Figura 2), faz-se necessário que o cliente envie ao SWIE uma requisição, utilizando o método POST, acompanhada de um conjunto de dados georreferenciados (latitude, longitude e value) e codificados no formato JSON. O parâmetro value é o valor da variável (temperatura, umidade relativa etc) que se deseja interpolar, e choice é o algoritmo que se deseja utilizar para a geração do modelo.

\footnotetext{
${ }^{1}$ https://play.google.com/store/apps/details?id=com.jualabs.aquaprev.app
} 
Recurso Model

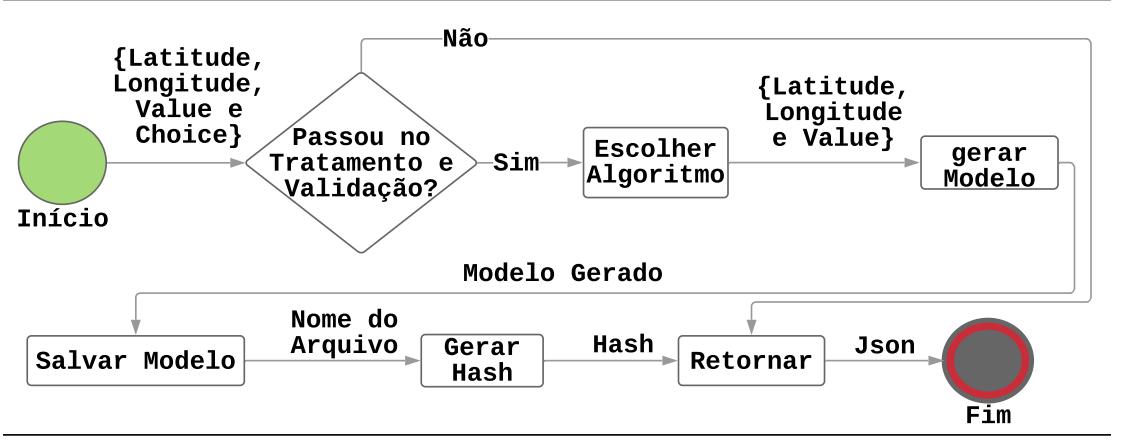

Figura 2. Fluxograma simplificado da execução do recurso Model Fonte: Próprio Autor

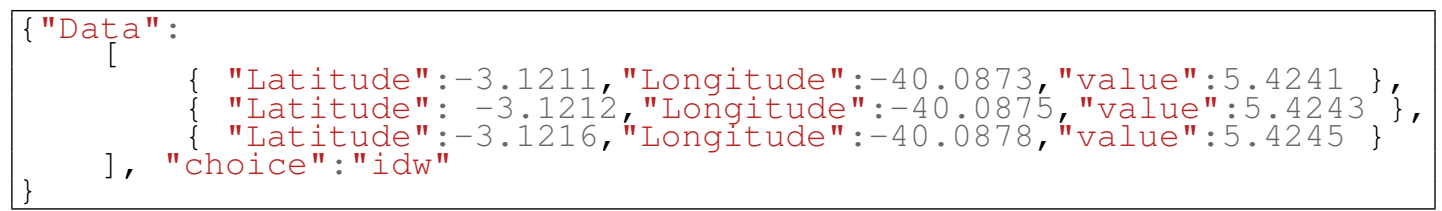

Figura 3. Exemplo de JSON enviado para geração do modelo por meio do recurso model.

Fonte: Próprio Autor

A versatilidade do SWIE está no fato de que ele independe da variável interpolada, permitindo o uso de variáveis contínuas de qualquer domínio. Além disso, o SWIE oferece três algoritmos para uso: Inverse Distance Weighted (IDW), Ordinary Kriging (OK) e Random Forest (RF). Enquanto IDW e OK são amplamente utilizados na interpolação de variáveis ambientais [Jakob and Young 2016, Lopes et al. 2017, De Vargas et al. 2019] e conhecidos por produzirem baixos erros de interpolação, o RF tem mostrado desempenho superior a esses em pesquisas recentes [Li et al. 2011, Appelhans et al. 2015, da Silva Júnior et al. 2019].

Na Figura 3 é exemplificado o formato JSON (JavaScript Object Notation) aceito pelo recurso Model. Nesse exemplo, Data é o parâmetro que representa o conjunto de dados georreferenciados (no exemplo são apenas 3 coordenadas). Além disso, há o parâmetro choice que informa qual é o algoritmo de interpolação escolhido para geração do modelo.

Os dados enviados ao recurso Model pelo cliente do SWIE passam por três validações: detecção de campos vazios no conjunto de dados, verificação da quantidade de dados recebidos e validação do algoritmo de interpolação escolhido. Como resultado desse recurso, é enviado ao cliente um documento JSON contendo o hash que identifica o modelo armazenado no sistema de arquivos do servidor onde o SWIE está sendo executado. O hash é composto pela codificação em base64 de uma string formada pelo timestamp da geração do modelo, utilizando data e hora no padrão POSIX time, concatenado ao tipo de algoritmo escolhido na geração do modelo. Desta forma, na chamada ao recurso interpolation, o cliente poderá fazer uso do modelo gerado anteriormente, passando o hash como parâmetro na requisição.

Na Figura 4 é mostrado o funcionamento da operação do recurso interpolation. Por meio de uma requisição usando o método POST, o cliente envia um arquivo JSON (exemplificado na Figura 5) indicando o modelo a ser utilizado na intepolação (hash) e as 
Recurso Interpolation

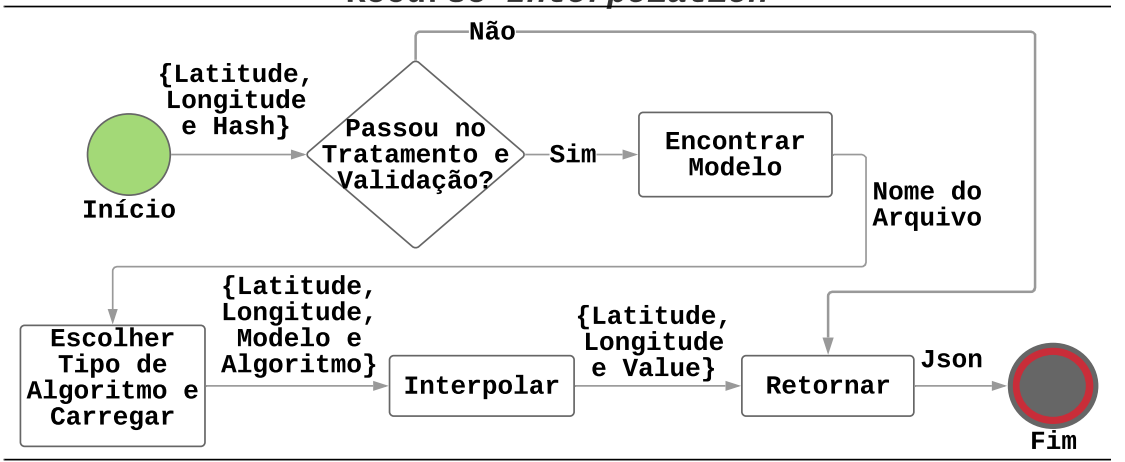

Figura 4. Fluxograma simplificado da execução do recurso Interpolation

Fonte: Próprio Autor

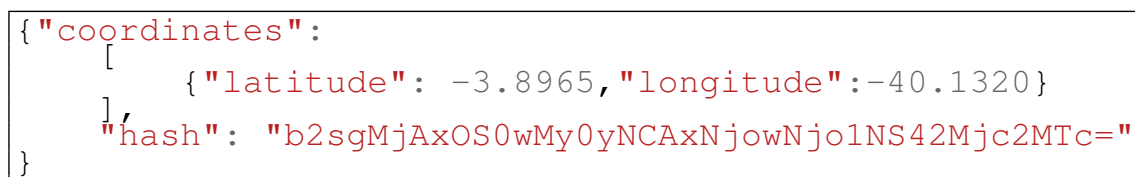

Figura 5. Exemplo de JSON de dados enviados por meio do recurso interpolation. Fonte: Próprio Autor

coordenadas geográficas (coordinates) de interesse. Esses dados serão usados para recuperar o modelo armazenado e realizar a interpolação propriamente dita. Com a conclusão da IE, o SWIE retorna para o cliente um conjunto de dados meteorológicos georreferenciados em formato JSON. Na Figura 6, observa-se o conjunto de dados retornado ao cliente, com as coordenadas enviadas e o valor interpolado correspondente.

Figura 6. Exemplo de JSON de dados retornado após a interpolação das coordenadas. Fonte: Próprio Autor

A escolha por separar a geração do modelo de sua efetiva utilização para interpolação foi definida para dar maior generalidade ao SWIE, tornando possível a reutilização de um modelo gerado repetidas vezes para diferentes conjuntos de pontos de interpolação por parte do cliente. Por isso, não é objetivo do SWIE a consulta aos hashes gerados, ficando a cargo dos clientes o gerenciamento e armazenamento dos hashes retornados pelo SWIE.

\subsection{Implementação do serviço}

Para a implementação do SWIE, optou-se pela utilização da linguagem $\mathrm{R}$ [R Core Team 2018] para computação estatística, por se tratar de linguagem open source, que garante um contínuo aprimoramento de bibliotecas e rotinas. Esta linguagem dispõe de pacotes para IE reconhecidos por sua estabilidade e confiabilidade, que já foram validados e aceitos pela comunidade acadêmica. Além de dispor de funções especiais para exibir, armazenar e organizar dados espaciais.

Utilizou-se da biblioteca R Plumber ${ }^{2}$ para implementação da interface REST do

\footnotetext{
${ }^{2}$ https://www.rplumber.io/
} 
SWIE, pois é uma alternativa nativa ao R que oferece uma rápida implantação do serviço, o que não ocorre em soluções alternativas. Também foi utilizada a biblioteca randomForest para geração e manipulação de modelos RF. Para OK e IDW, fez-se uso do pacote gstat. A escolha dessas bibliotecas deve-se a sua ampla utilização em trabalhos na área [da Silva Júnior et al. 2019, Abreu 2016, CHAGAS et al. 2015].

Para o armazenamento de valores específicos do ambiente do sistema, utilizou-se o pacote config], o qual facilita no gerenciamento de valores de configuração específicos do ambiente, como é o caso do uso de valores distintos para ambientes de desenvolvimento, teste e produção. Já para a realização da interpolação, fez-se uso da função predict, presente no pacote stats. A escolha por essa biblioteca se deu por se tratar de uma biblioteca genérica para predições relacionadas a qualquer tipo de modelo.

\section{Avaliação de desempenho do SWIE}

A avaliação de desempenho buscou observar o comportamento do SWIE desenvolvido sob diferentes condições, de modo a identificar gargalos no serviço. Vale salientar que este trabalho não avaliou a acurácia dos algoritmos, visto que esse fator tem forte relação com a variável interpolada e já objeto de trabalhos específicos desenvolvidos por outros autores [da Silva Júnior et al. 2019, Li and Heap 2014, Xavier et al. 2016].

A avaliação foi executada como um experimento completamente fatorial, onde cada requisição foi tratada como uma execução independente. A medida de desempenho avaliada foi o tempo de resposta da requisição, computada como o tempo despendido desde o disparo da requisição pelo cliente, o processamento desta até o retorno ao cliente. Utilizou-se, nesta análise, a média do tempo de resposta das requisições.

O experimento assume que um determinado número de usuários virtuais realiza requisições simultaneamente, a fim de aumentar a demanda por recursos do servidor. Cada usuário virtual envia ao SWIE, sequencialmente, 500 requisições. Esse parâmetro foi calculado a partir de amostras do tempo de resposta colhidas em dois experimentos prévios, considerando um erro de amostragem de 1\% e 95\% de confiança [Jain 1990].

O número de coordenadas geográficas (latitude e longitude) enviadas por requisição do tipo interpolation fora fixado em 1, para emular o cenário de pior caso em que os usuários requisitam cada localização em uma requisição. As coordenadas foram geradas pela feramenta Random Point Generator ${ }^{4}$ considerando o território brasileiro.

Para a realização dos testes, utilizou-se a ferramenta Apache JMeter ${ }^{5}$, que é um software open source desenvolvido em Java para a realização de testes de estresse e desempenho em sistemas distribuídos [de Sousa Santos and dos Santos Neto 2008]. Para eliminar impactos causados pela rede no desempenho do serviço, o SWIE e a ferramenta de medição JMeter foram hospedados em um mesmo computador, com processador Intel Xeon com 24 núcleos de processamento, 16 GB de memória RAM, 1 TB de armazenamento e sistema operacional Linux.

O plano de teste empregou os fatores e níveis apresentados na Tabela 1 . A quantidade de usuários virtuais variou em 1, 5, 25 e 50. Pretendeu-se, com isso, determinar a

\footnotetext{
${ }^{3}$ https://cran.r-project.org/web/packages/config/vignettes/introduction.html

${ }^{4} \mathrm{http}: / / \mathrm{www} . \mathrm{geomidpoint} . \mathrm{com} / \mathrm{random} /$

${ }^{5}$ https://jmeter.apache.org/
} 
quantidade limite de usuários que uma instância do serviço, sobre o hardware utilizado, pode suportar. Já o fator Mix Modelo/Interpolação determina a proporção das requisições executadas para geração do modelo e para interpolação. Cada nível indica o percentual de requisições feitas ao recurso model e a porcentagem de requisições ao recurso interpolation, respectivamente. Assim, nos experimentos realizados para o nível 0\%/100\%, por exemplo, contou-se apenas com a execução de requisições do tipo interpolation.

\begin{tabular}{|l|l|}
\hline Fatores & Níveis \\
\hline Usuários Virtuais & $1,5,25$ e 50 \\
\hline Mix Modelo/Interpolação & $0 \% / 100 \%, 25 \% / 75 \%, 50 \% / 50 \%, 75 \% / 25 \%$ e $100 \% / 0 \%$ \\
\hline Pontos do Modelo & $100,200,300,400$ e 500 \\
\hline Algoritmos & IDW, OK e RF \\
\hline
\end{tabular}

Tabela 1. Fatores utilizados no experimento

Fonte: Próprio Autor

O fator Pontos do Modelo refere-se à quantidade de pontos enviados nas requisições do recurso model, ou seja, o número de amostras espaciais da variável ambiental sob estudo. Esse fator, variou de 100 a 500 pontos, em intervalos de 100. Desta forma, foi possível observar como a quantidade de dados submetidos para a geração do modelo, operação de maior impacto no serviço, afeta o desempenho do serviço. Os pontos utilizados na geração dos modelos são amostras reais de evapotranspiração de referência diária obtidas a partir de estações meteorológicas do Instituto Nacional de Meteorologia.

Por fim, o fator Algoritmos representa o algoritmo demandado nas requisições de geração do modelo, sendo escolhido um dos 3 algoritmos implementados no SWIE: IDW, OK e RF. O objetivo desse fator é observar como a escolha do algoritmo impacta no tempo de geração do modelo e na IE a partir do modelo gerado. Note-se que, dentro de um experimento, todas as requisições utilizam o mesmo algoritmo.

A partir dos resultados, buscou-se entender qual a relação e impacto estabelecidos entre os fatores e o tempo de resposta do SWIE. A análise estatística para determinar a presença de interações significativas em relação ao tempo de resposta, baseou-se no teste ANOVA e análise gráfica [Jain 1990].

\section{Resultados}

No diagrama de caixa (Figura 7a) é mostrado como o tempo de resposta se comporta frente a variações no número de usuários virtuais. É possível perceber que o aumento do número de usuários virtuais tem pequeno impacto sobre a mediana do tempo de resposta, indicando que, para a maior parte das requisições, o tempo de resposta é baixo. Contudo, observa-se um forte impacto na variabilidade do tempo de resposta, já que, para mais de 25 usuários, obtiveram-se tempos de resposta com mais de 10 segundos.

O aumento do tempo de resposta, contudo, deve-se muito mais ao fator Algoritmos, cujo resultado é mostrado na Figura $7 \mathrm{~b}$. Pode-se verificar que o tempo de resposta é claramente impactado pela escolha do algoritmo de interpolação. Enquanto os algoritmos IDW e OK apresentaram tempos de resposta baixos (mediana de aproximadamente $380 \mathrm{~ms}$ para o IDW e $541 \mathrm{~ms}$ para OK), o algoritmo RF apresentou mediana superior a 10 segundos, alcançando, inclusive, valores maiores do que 50 segundos (máximo de 57 segundos). Esse resultado corrobora com o observado em [da Silva Júnior et al. 2019], o 


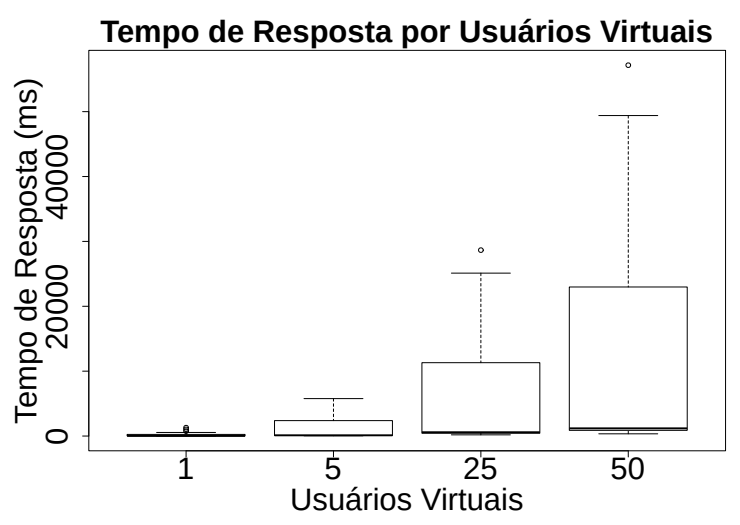

(a) Usuários virtuais

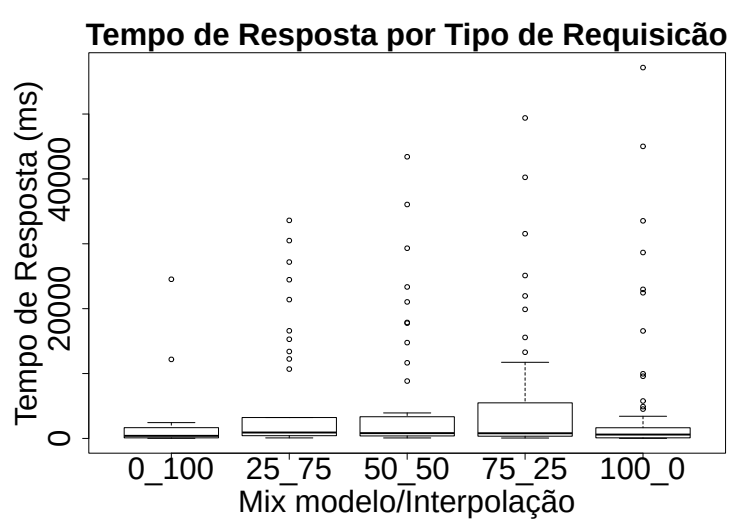

(c) Tipo de requisição

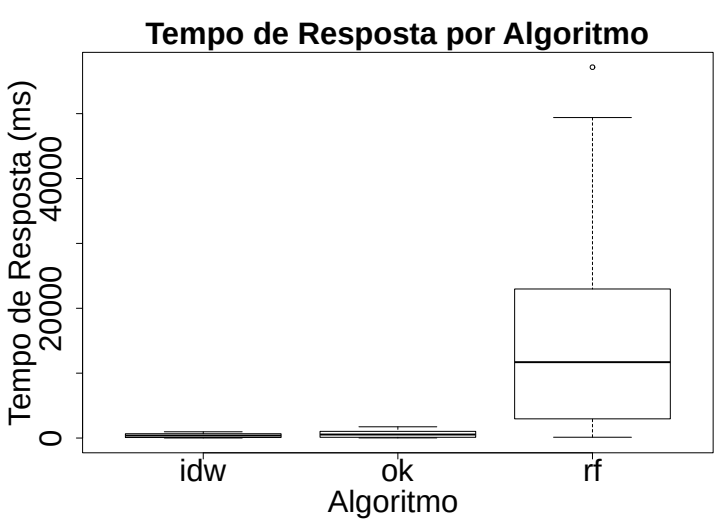

(b) Algoritmo de interpolação

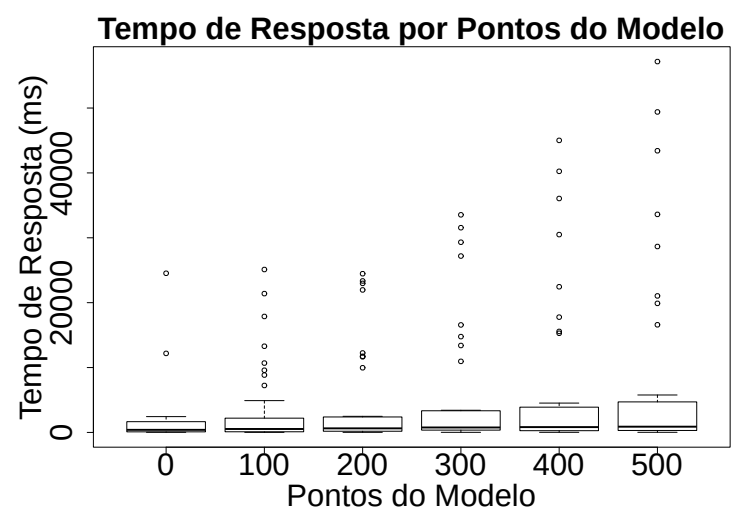

(d) Pontos do modelo

Figura 7. Tempo de resposta do serviço de acordo com: (a) usuários virtuais; (b) algoritmo de interpolação; (c) tipo de requisição (mix modelo/interpolação); (d) pontos do modelo.

qual mostrou um tempo médio de resposta do RF, aproximadamente 8 vezes maior do que o apresentado pelo algoritmo OK.

Também se observa grande variabilidade no tempo de resposta do algoritmo RF. Tal variabilidade está associada à forma como esse algoritmo trabalha, já que esse é um interpolador que constrói um conjunto de árvores de decisão, o que pode demandar grande esforço computacional, principalmente nos casos em que há um maior número de usuários interagindo com o serviço.

Isto pode ser melhor observado na Figura 8 , que mostra a interação entre os fatores Usuários Virtuais e Algoritmos sobre o tempo de resposta. Pode-se inferir que os tempos de resposta mais altos do algoritmo RF têm relação com o número de Usuários Virtuais, pois quando a quantidade de usuários é mais baixa (1 e 5), o tempo médio de resposta é baixo para todos os algoritmos avaliados. Já nos casos de 25 e 50 usuários virtuais, o algoritmo RF obtém pior performance e os demais algoritmos mantêm-se muito próximos.

Na Figura 7c são apresentados os resultados para o fator Mix modelo/interpolação. No eixo das abscissas, o par 0_100 corresponde ao nível 0\%/100\%, e os demais seguem esta ideia. Pode-se observar um aumento da variabilidade do tempo de resposta, com consequente aumento da média em cada caso, do nível 0\%/100\% (média de $3.5 \mathrm{~s}$ ) ao nível $25 \% / 75 \%$ (média de $5.5 \mathrm{~s}$ ), decorrente do fato de que a geração dos modelos possui, co- 


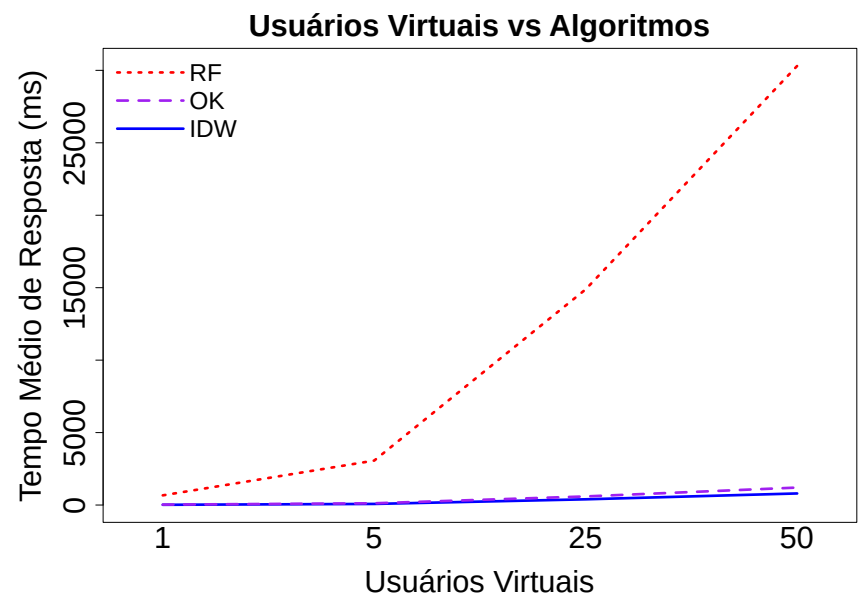

Figura 8. Interação entre a quantidade de Usuários Virtuais e os Algoritmos no tempo Fonte: Próprio Autor

mumente, maior complexidade computacional, e assim, um maior número de requisições para geração de modelos, exigirá maior tempo de processamento.

Por outro lado, observa-se uma diminuição do tempo de resposta quando se passa para o nível 100\%/0\% (média de $4.8 \mathrm{~s}$ ), se aproximando do encontrado no nível 0\%/100\%. Isso acontece porque a mudança na proporção de requisições, impacta, principalmente, nos tipos de operações que são feitas em disco. No SWIE todos os modelos são armazenados diretamente em disco, portanto, a mudança no mix altera diretamente a quantidade de operações de escrita/leitura em disco. Os casos que fazem o mesmo tipo de operação, somente leitura (nível 0\%/100\%) e somente escrita (100\%/0\%), promovem um melhor desempenho, devido à maior liberdade que o sistema operacional e a controladora de disco têm em otimizar suas operações, sendo a operação de escrita mais lenta do que a de leitura (e por isso a maior média no nível 0\%/100\%).

Nos casos intermediários, o aumento da quantidade de operações de escrita, em comparação com o caso $0 \% / 100 \%$, leva a um aumento gradativo do tempo de resposta atingindo, de modo geral, valores médios maiores, até mesmo do que o nível 100\%/0\%. Isso é decorrente da menor liberdade para otimização que o sistema operacional e a controladora de disco têm para execução das operações de leitura e escrita.

Na Figura 7d, ilustra-se a relação do tempo de resposta com o fator Pontos do Modelo. O nível com 0 pontos indica os casos onde só há requisições para interpolação, sendo equivalente ao Mix Modelo/Interpolação 0\%/100\%, já discutido. Pode-se observar, nesse resultado, que o tempo de resposta aumenta ligeiramente ao variar-se o número de pontos do modelo, saindo de uma mediana de $531 \mathrm{~ms}$ com 100 pontos e atingindo uma mediana de 885 ms com 500 pontos. O que é esperado, já que o custo computacional desses algoritmos é relacionado com a quantidade de pontos passados como entrada.

Para avaliar a influência estatística de cada fator sobre o tempo de resposta recorreu-se ao ANOVA, cujos resultados estão na Tabela 2. Para cada fator são demonstrados a variação explicada (coluna SQ), sua respectiva contribuição percentual, o valor da estatística de teste F (coluna Valor F) e o p-valor, que indica a probabilidade da evidência contra a hipótese nula, de que não há impacto do fator sobre o tempo de resposta.

Considerando nível de confiança de 95\%, conclui-se que há uma associação esta- 


\begin{tabular}{|l|l|l|l|l|}
\hline Fator & SQ & Contribuição \% & Valor F & p-valor \\
\hline Mix Modelo/Interpolação & 91395282 & 0,41 & 0,503 & 0,733 \\
\hline Algoritmos & 9366991365 & 42,34 & 103,133 & 0,0000000 \\
& & & & 000000002 \\
\hline Pontos do Modelo & 555080773 & 2,51 & 3,056 & 0,018 \\
\hline Usuários Virtuais & 3435880804 & 15,53 & 25,22 & 0,0000000 \\
& & & & 000000863 \\
\hline Resíduos & 8673712530 & - & - & - \\
\hline
\end{tabular}

Tabela 2. Resultado da análise de variância (ANOVA)

Fonte: Próprio Autor

tisticamente significativa, entre o tempo de resposta e os fatores Usuários Virtuais e Pontos do Modelo. Contudo, nota-se que o fator Pontos do Modelo traz menor impacto ao tempo de resposta (contribuição de $2,51 \%$ ), reafirmando o que já fora observado através dos diagramas de caixa. Os resultados para o fator Mix Modelo/Interpolação apontam fraca influência desse sobre o tempo de resposta.

\section{Conclusão}

A importância deste estudo está na apresentação de um serviço web escalável para interpolação espacial, que se utiliza do estilo arquitetônico REST. O serviço contribui diretamente para o desenvolvimento de aplicações, já que pode torná-las mais robustas no atendimento de usuários em larga escala.

Tal serviço foi concebido para ser também versátil, permitindo a interpolação de qualquer variável contínua e implementando três diferentes algoritmos de interpolação espacial. Desta forma, abre-se um leque de possibilidades no desenvolvimento de soluções digitais, já que essas novas soluções poderão reutilizar a interpolação como serviço, diminuindo seu tempo de desenvolvimento.

Neste trabalho pôde-se avaliar o desempenho do SWIE a partir de um experimento completamente fatorial, levando em conta múltiplos fatores que afetam o desempenho do serviço. De modo geral, os algoritmos clássicos - OK e IDW - apresentaram um menor tempo de resposta se comparados ao algoritmo RF. Esse resultado indica que deve haver um compromisso entre desempenho e acurácia na escolha do algoritmo para interpolação, pois enquanto o algoritmo RF tem obtido melhor acurácia do que os métodos convencionais [da Silva Júnior et al. 2019], seu uso tem alto custo computacional. Assim, a busca por uma maior precisão deve vir acompanhada de uma cuidadosa implantação do serviço de interpolação - utilizando técnicas como replicação e balanceamento de carga - para que o usuário do serviço não venha a sofrer com o desempenho do algoritmo RF.

Como limitação do serviço, pode-se destacar que esse é baseado exclusivamente na latitude e longitude. Esta limitação pode tornar o serviço inapropriado para interpolar variáveis que sejam dependentes de outras variáveis, tal como a precipitação cuja interpolação é melhorada ao se considerar aspectos como relevo e cobertura do solo, por exemplo [Goovaerts 2000]. Como trabalho futuro, pretende-se sobrepujar esta limitação estendendo o serviço pelo emprego de métodos de interpolação que permitam o uso de variáveis auxiliares [Li and Heap 2008]. Além disso, almeja-se adicionar outros algoritmos de interpolação espacial para aumentar a versatilidade do serviço. 


\section{Referências}

[Abreu 2016] Abreu, J. M. F. (2016). Modelos de Data Mining para suporte a avaliações sensoriais do vinho. $\mathrm{PhD}$ thesis, Instituto Superior de Engenharia do Porto.

[Appelhans et al. 2015] Appelhans, T., Mwangomo, E., Hardy, D. R., Hemp, A., and Nauss, T. (2015). Evaluating machine learning approaches for the interpolation of monthly air temperature at Mt. Kilimanjaro, Tanzania. Spatial Statistics, 14:91-113.

[CHAGAS et al. 2015] CHAGAS, C. d. S., CARVALHO JUNIOR, W. d., Bhering, S. B., and Pereira, N. R. (2015). Mapeamento digital da ctc em solos do semiárido brasileiro. In Embrapa Solos-Artigo em anais de congresso (ALICE). In: CONGRESSO BRASILEIRO DE CIÊNCIA DO SOLO, 35., 2015, Natal. O solo e suas ....

[da Silva Júnior et al. 2019] da Silva Júnior, J. C., Medeiros, V., Garrozi, C., Montenegro, A., and Gonçalves, G. E. (2019). Random forest techniques for spatial interpolation of evapotranspiration data from brazilian's northeast. Computers and Electronics in Agriculture, 166:105017.

[de Sousa Santos and dos Santos Neto 2008] de Sousa Santos, I. and dos Santos Neto, P. d. A. (2008). Automação de testes de desempenho e estresse com o jmeter.

[De Vargas et al. 2019] De Vargas, T., Gomes, M. G., Belladona, R., and Adami, M. V. D. (2019). Aplicação do interpolador idw para elaboração de mapas hidrogeológicos paramétricos na região da serra gaúcha. Scientia cum Industria, 6(3):38-43.

[Goovaerts 2000] Goovaerts, P. (2000). Geostatistical approaches for incorporating elevation into the spatial interpolation of rainfall. Journal of hydrology, 228(1-2):113-129.

[Jain 1990] Jain, R. (1990). The art of computer systems performance analysis: techniques for experimental design, measurement, simulation, and modeling. John Wiley \& Sons.

[Jakob and Young 2016] Jakob, A. A. E. and Young, A. F. (2016). O uso de métodos de interpolação espacial de dados nas análises sociodemográficas. Anais, pages 1-22.

[Li and Heap 2008] Li, J. and Heap, A. D. (2008). A review of spatial interpolation methods for environmental scientists. Geoscience Australia, Canberra. OCLC: 312275544.

[Li and Heap 2014] Li, J. and Heap, A. D. (2014). Spatial interpolation methods applied in the environmental sciences: A review. Environmental Modelling \& Software, 53:173189.

[Li et al. 2011] Li, J., Heap, A. D., Potter, A., and Daniell, J. J. (2011). Application of machine learning methods to spatial interpolation of environmental variables. Environmental Modelling \& Software, 26(12):1647-1659.

[Lopes et al. 2017] Lopes, I., De Melo, J. M. M., and Leal, B. G. (2017). Espacialização da temperatura do ar para a região do submédio são francisco. Irriga, 22(1):177.

[R Core Team 2018] R Core Team (2018). R: A Language and Environment for Statistical Computing. R Foundation for Statistical Computing, Vienna, Austria.

[Xavier et al. 2016] Xavier, A. C., King, C. W., and Scanlon, B. R. (2016). Daily gridded meteorological variables in Brazil (1980-2013). International Journal of Climatology, 36(6):2644-2659. 